\title{
Behavioral changes and growth deficits in a CRISPR engineered mouse model of the schizophrenia-associated $3 q 29$ deletion
}

\author{
Timothy P. Rutkowski ${ }^{1}$. Ryan H. Purcell ${ }^{2}$ Rebecca M. Pollak $\mathbb{D}^{1} \cdot$ Stephanie M. Grewenow ${ }^{1} \cdot$ Georgette M. Gafford ${ }^{1}$. \\ Tamika Malone $^{1}$ - Uswa A. Khan ${ }^{1}$ - Jason P. Schroeder ${ }^{1}$. Michael P. Epstein ${ }^{1} \cdot$ Gary J. Bassell $^{2} \cdot$ Stephen T. Warren $^{1}$. \\ David Weinshenker ${ }^{1} \cdot$ Tamara Caspary $^{1} \cdot$ Jennifer Gladys Mulle ${ }^{1}$
}

Received: 26 November 2018 / Revised: 13 February 2019 / Accepted: 18 March 2019 / Published online: 11 April 2019

(c) Springer Nature Limited 2019

\begin{abstract}
The 3q29 deletion confers increased risk for neuropsychiatric phenotypes including intellectual disability, autism spectrum disorder, generalized anxiety disorder, and a $>40$-fold increased risk for schizophrenia. To investigate consequences of the 3q29 deletion in an experimental system, we used CRISPR/Cas9 technology to introduce a heterozygous deletion into the syntenic interval on C57BL/6 mouse chromosome 16. mRNA abundance for 20 of the 21 genes in the interval was reduced by $\sim 50 \%$, while protein levels were reduced for only a subset of these, suggesting a compensatory mechanism. Mice harboring the deletion manifested behavioral impairments in multiple domains including social interaction, cognitive function, acoustic startle, and amphetamine sensitivity, with some sex-dependent manifestations. In addition, 3q29 deletion mice showed reduced body weight throughout development consistent with the phenotype of 3q29 deletion syndrome patients. Of the genes within the interval, $D L G 1$ has been hypothesized as a contributor to the neuropsychiatric phenotypes. However, we show that $\mathrm{Dlg}^{+/-}$mice did not exhibit the behavioral deficits seen in mice harboring the full 3q29 deletion. These data demonstrate the following: the 3q29 deletion mice are a valuable experimental system that can be used to interrogate the biology of 3q29 deletion syndrome; behavioral manifestations of the 3q29 deletion may have sex-dependent effects; and mouse-specific behavior phenotypes associated with the 3q29 deletion are not solely due to haploinsufficiency of $\mathrm{D} \lg 1$.
\end{abstract}

\section{Introduction}

The 3 q29 deletion syndrome is caused by heterozygous deletion of a $1.6 \mathrm{Mb}$ interval containing 21 protein-coding genes. Individuals with the deletion are at increased risk for intellectual disability, autism spectrum disorder (ASD),

These authors contributed equally: David Weinshenker, Tamara Caspary, Jennifer Gladys Mulle

Supplementary information The online version of this article (https:// doi.org/10.1038/s41380-019-0413-5) contains supplementary material, which is available to authorized users.

Jennifer Gladys Mulle

jmulle@emory.edu

1 Department of Human Genetics, Emory University School of Medicine, Atlanta, GA 30322, USA

2 Department of Cell Biology, Emory University School of Medicine, Atlanta, GA 30322, USA generalized anxiety disorder, and schizophrenia (SZ) [1-7]. In the largest association study of copy number variation and SZ to date, the 3q29 deletion emerges as one of only eight loci achieving genome-wide significance [3]. Separate analyses have shown that the 3q29 deletion confers an exceptional risk for SZ, with an estimated effect size of $>40$ $[8,9]$. This large effect size, coupled with the relatively low complexity of the interval and the high synteny between the human and mouse genomes, make this variant an ideal candidate for the development of a mouse experimental system. This is further aided by CRISPR/Cas9 technology facilitating engineered genomic rearrangements [10]. Collectively, the $3 \mathrm{q} 29$ deletion is a compelling region to study because it provides an opportunity for genetic dissection of complex neuropsychiatric phenotypes.

Of the 21 genes within the interval, $D L G 1$ and PAK2 have received attention as attractive candidates contributing to neuropsychiatric phenotypes [11]. DLG1 (a.k.a. SAP97) is a scaffolding protein that interacts with $N$-methyl-D-aspartate (NMDA) and $\alpha$-amino-3-hydroxyl-5-methyl-4-isoxazole- 
propionate (AMPA) type glutamate receptors [12, 13]. Suggestive evidence for association between $D L G 1$ and neuropsychiatric phenotypes comes from sequencing studies where SZ cases are enriched for $D L G 1$ variants compared with controls $[14,15]$. An expression study of post-mortem tissue found decreased DLG1 in the prefrontal cortex in SZ patients [16]. PAK2 is implicated by its function as a regulator of cytoskeletal dynamics. Based on the current evidence for their possible involvement in neuropsychiatric phenotypes, mouse models have been created for both Dlg1 and Pak2 deficiency. A brain-specific conditional Dlg1 deletion mouse displays some sex-specific behavioral phenotypes, including subtle cognitive and motor deficits [17]. A $\mathrm{Pak2}^{+/}$mouse model displays autism-related behaviors and neuronal deficits [18]. Drosophila models also support involvement of $d l g$ and pak, but behavioral and molecular phenotypes are only seen when $d l g$ and $p a k$ are simultaneously reduced [19].

We undertook a two-stepapproach to better understand the neuropsychiatric consequences of the $3 \mathrm{q} 29$ deletion and the possible contribution of $D L G 1$ to these phenotypes. First, we created a mouse harboring a heterozygous 3q29 deletion (henceforth referred to as B6.Del16 ${ }^{+/ B d h l-T f r c}$ mice [nomenclature adapted from Mouse Genome Informatics, MGI:6241487]), and assessed gene expression, protein abundance, developmental weight trajectories, and behavioral changes associated with the deletion. Second, we used $D \lg 1^{+/}$mice to test the hypothesis that DLGl haploinsufficiency alone is sufficient to manifest B6.Del16 (Bdhl-Tfrc $_{-}$ associated phenotypes. We performed parallel analyses in both B6.Del16 $6^{+B d h l-T f r c}$ and $D l g 1^{+-}$mice. Our results allow for integration of existing data from mouse models of other 3q29 interval genes and pave the way for careful and rigorous dissection of the genetic drivers for neuropsychiatric phenotypes within the 3q29 interval.

\section{Materials and methods}

See Supplemental Material for detailed Methods and Protocols.

\section{Mouse strains and alleles}

All mouse work was performed under the approved guidelines of the Emory University IACUC. To generate the 3 q29 deletion in the mouse, two clustered regularly interspaced short palindromic repeat (CRISPR) guide RNAs (gRNAs) were designed at the syntenic loci in the mouse genome. The Emory Transgenic and Gene Targeting core injected $50 \mathrm{ng} / \mu \mathrm{l}$ of each gRNAs and $100 \mathrm{ng} / \mu \mathrm{l}$ Cas9 RNA into single-cell C57BL/6N zygotes. Embryos were cultured overnight and transferred to pseudopregnant females.
Resulting pups were screened for the deletion via PCR and for an absence of genomic rearrangements by Southern blot (see Supplemental Materials for primer sequences used for genotyping and probe details). The founders used (\#127, \#131) were backcrossed to C57BL/6N and analysis commenced in the $\mathrm{N} 4$ generation. All mice were maintained on a C57BL/6N background sourced from Charles River Laboratories. The $\mathrm{Dlg} \mathrm{I}^{+/-}$mice [MGI:3699270] [20] were obtained from Dr. Jeffrey Miner (Washington University in St Louis) on a mixed 129/C57BL/6J background. The mice were backcrossed using marker-assisted breeding (DartMouse $^{\mathrm{TM}}$, https://geiselmed.dartmouth.edu/dartmouse/) to obtain N6 Dlg1 ${ }^{+-}$mice that were $99 \%$ congenic on a C57BL/6N background (henceforth referred to as B6. $D \lg 1^{+-}$). Number of animals used in experiments is indicated in figure legends.

\section{Gene and protein expression in mouse brain tissue}

RNA was isolated from forebrain samples from 16- to 20week-old mice ( $N=4$ for each genotype) and gene expression was measured by real-time quantitative PCR (three technical replicates per sample). See Supplemental Table 1 for list of Taqman assay IDs. Protein was isolated from forebrain samples from 16- to 20-week-old mice according to standard procedures and protein levels were measured by western blot. See Supplemental Table 2 for antibodies.

\section{Behavior tests}

Mice were on a 12-h light/dark cycle and were given food and water ad libitum. Mice were between the ages of 16 and 20 weeks when behavioral testing commenced. All equipment was cleaned using Virkon. No method of randomization was utilized in the behavior studies.

\section{Social interaction and Morris water maze}

The three-chambered social interaction paradigm was adapted from Yang et al. [21]. The Morris water maze (MWM) was conducted using the same paradigm as Chalermpalanupap et al. [22].

\section{Prepulse inhibition (PPI)}

The San Diego Instruments (La Jolla, CA) SR-LAB startle response system was used to perform PPI. We used a 2-day paradigm. On day 1 , we tested the ability of the mouse to startle to a series of increasing tones. On day 2, we subjected each mouse through the PPI paradigm. PPI was calculated as a percentage using the following equation: (startle-startle.PP/startle) $\times 100$. 


\section{Amphetamine-induced locomotor activity}

The assay was performed in a locomotor chamber (San Diego Instruments), which consisted of a plexiglass cage $(48 \times 25 \times 22 \mathrm{~cm})$ containing corncob bedding that rested between an apparatus containing infrared beams. The mice were given an injection of either saline or amphetamine (2.5 or $7.5 \mathrm{mg} / \mathrm{kg}$, i.p.), and post-injection ambulations were measured for $2 \mathrm{~h}$. All treatments were spread over 3 weeks and were counter balanced such that not all of the mice received the same injection in a given week. For the $7.5 \mathrm{mg} / \mathrm{kg}$ amphetamine dose, a subset of mice was video recorded between the 30 and 60 min post-injection timepoint. These videos were scored for stereotypy using the criteria as described previously [23] by an experimenter blinded to genotype.

\section{Additional measures}

Details on paradigms for circadian rhythm, elevated plus maze, open field, marble burying, social interaction, PPI, fear conditioning, and histology can be found in Supplemental Materials. Sample sizes of 8-16 mice per sex and genotype were used in agreement with existing literature.

\section{Analysis}

\section{Gene expression}

Data were analyzed by two-way, repeated-measures analysis of variance (ANOVA) followed by multiple comparisons with Sidak's correction, where appropriate (i.e., only when a significant $[p<0.05]$ genotype effect or interaction was detected).

\section{Behavioral tests}

All behavior tests were analyzed following these parameters unless otherwise specified. Behavior was analyzed by unpaired $t$-test (when comparing two groups) or two-way, repeated-measures ANOVA (when comparing $>2$ groups), followed by multiple comparisons with Sidak's correction where appropriate (i.e., only when a significant $[p<0.05]$ genotype effect or interaction was detected).

\section{Amphetamine-induced locomoter activity}

All analyses performed in R [24]. Because the ambulation data were not normally distributed, the inverse normal function was used to transform the data to an approximately normal distribution. Proper transformation of the data was confirmed with the Shapiro-Wilk normality test implemented using the stats package [24]. Linear mixed-effects models with restricted maximum likelihood estimation were implemented using the lme4 package [25]. All models included subject ID and timepoint as random effects; males and females were analyzed separately. Models tested for the main effects of genotype and treatment (saline, $2.5 \mathrm{mg} / \mathrm{kg}$ amphetamine, and $7.5 \mathrm{mg} / \mathrm{kg}$ amphetamine), and for an interaction between genotype and treatment. Saline was set as the reference treatment and WT was set as the reference genotype for all analyses. $p$-Values were calculated using Satterthwaite's method using the lmerTest package [26].

\section{Acoustic startle and growth curves}

Analyses for acoustic startle and growth curves were performed in R [24]. Additional details on the analyses for these tasks can be found in the supplemental materials. All data represented as mean \pm SEM.

\section{Results}

\section{Confirmation of deletion using CRISPR/Cas9}

To generate mice that recapitulated the $3 \mathrm{q} 29$ deletion, we took advantage of several features of the syntenic region on mouse chromosome 16. The mouse and human regions are almost identical with all 21 genes present in the same order (Bex6 present in the mouse, not human). The mouse syntenic region is inverted; inversion breakpoints are identical to the SZ and ID-associated deletion breakpoints. The mouse region is also slightly smaller compared with the human region, 1.26 vs. $1.6 \mathrm{Mb}$ (Fig. 1a). To generate B6.Del16 ${ }^{+/ B d h l-T f r c}$ mice, we mimicked the 3q29 deletion breakpoints by designing CRISPR gRNAs proximal to Bdhl and distal to Tfrc (Fig. 1b). We performed PCR to confirm the presence of the heterozygous deletion in potential founder animals. To investigate whether the double-strand breaks during CRISPR/ Cas9 editing created additional genomic rearrangements in the region, we performed a Southern blot using a probe centromeric to the proximal breakpoint (Fig. 1b). The radi-

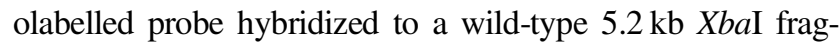
ment on the intact, non-deleted chromosome and a $6.2 \mathrm{~kb}$ $X b a \mathrm{I}$ fragment on the deleted chromosome. Of the 23 potential founders, we identified seven (30\%) animals that appeared to be properly targeted. The PCR products across the deletion were sequenced to determine the precise breakpoints for the two founders used [\#127 and \#131] (see Supplemental Materials).

We analyzed the expression of the 19/22 genes (Slc51a and Zdhhc19 are not expressed in brain; Bex6 is mousespecific and not present in the human interval) using forebrain tissue from both adult female and male B6. Del16 ${ }^{+/ B d h l-T f r c}$ mice. Using real-time PCR (RT-PCR), we 
a

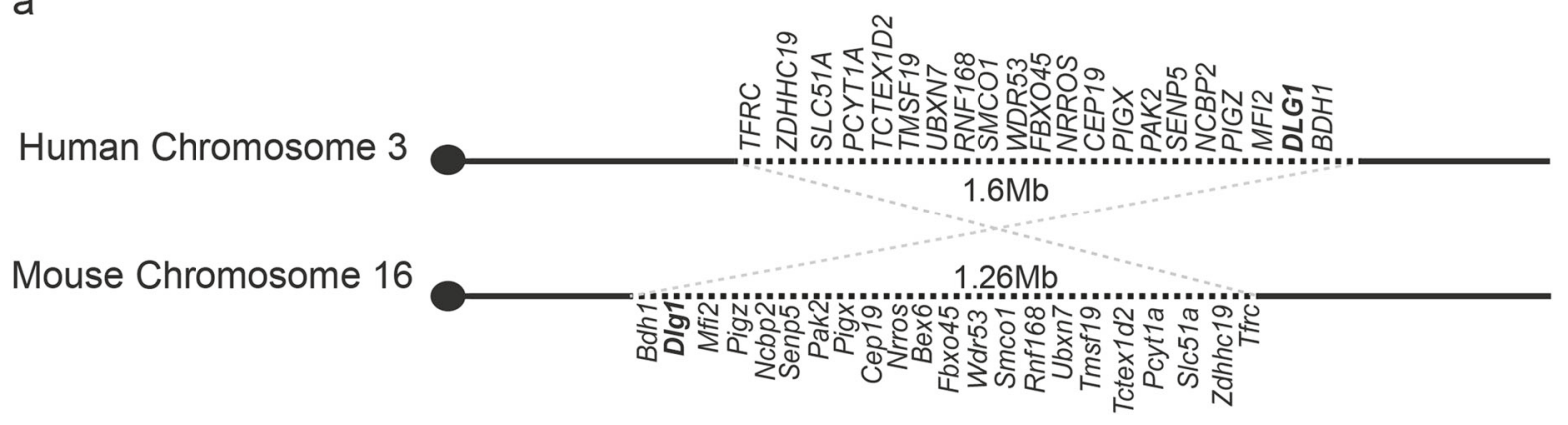

b

$\begin{aligned} & \text {.... 3q29 Interval }(1.26 \mathrm{Mb}) \\ & \text { C } \\ & \text { CRISPR-A } \\ &(16: 31,369,117 \\ & \text { CRISPR-B } \\ &(16: 32,634,414 \\ & \square \text { Southern Probe } \\ & X=X b a l \\ & \rightarrow \text { Forward Primer } \\ & \leftarrow \text { Reverse Primer }\end{aligned}$
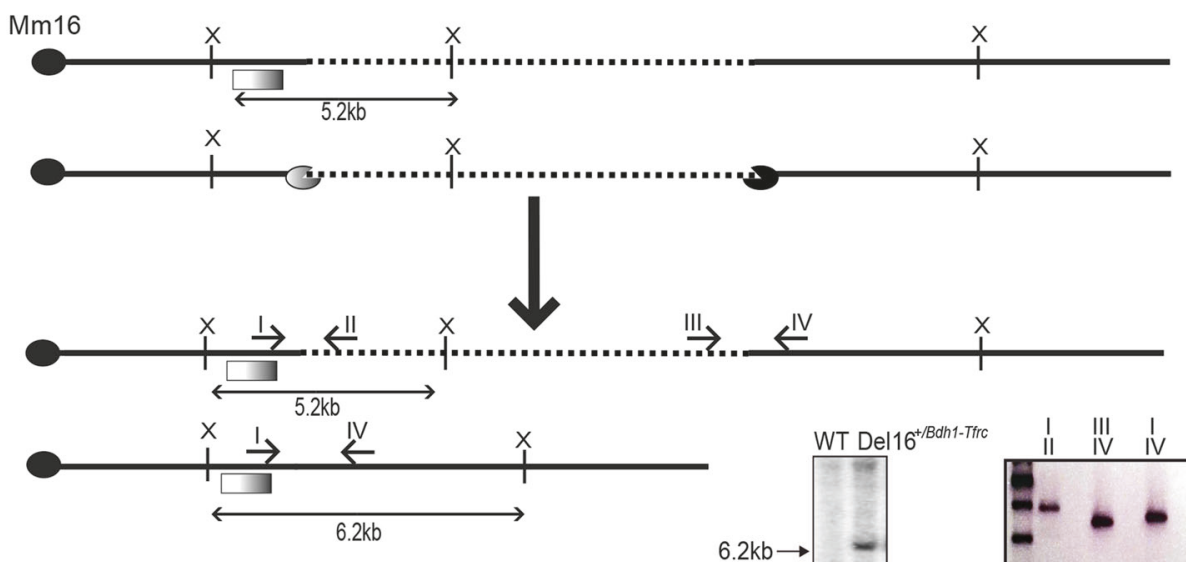

B6.WT Males $\square$ B6.Del16 $6^{+/ B d h 1-T f r}$ Males

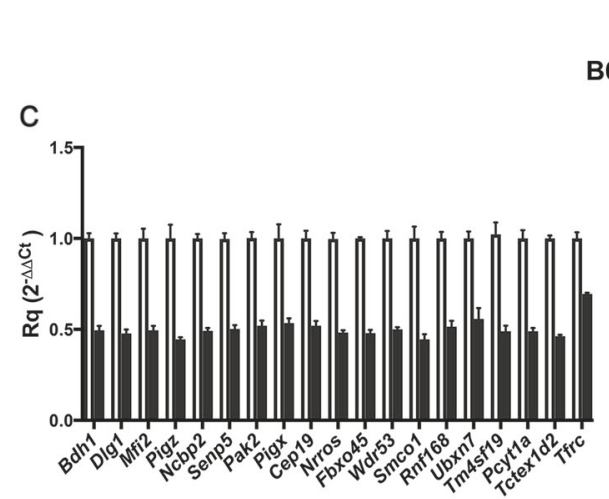

d
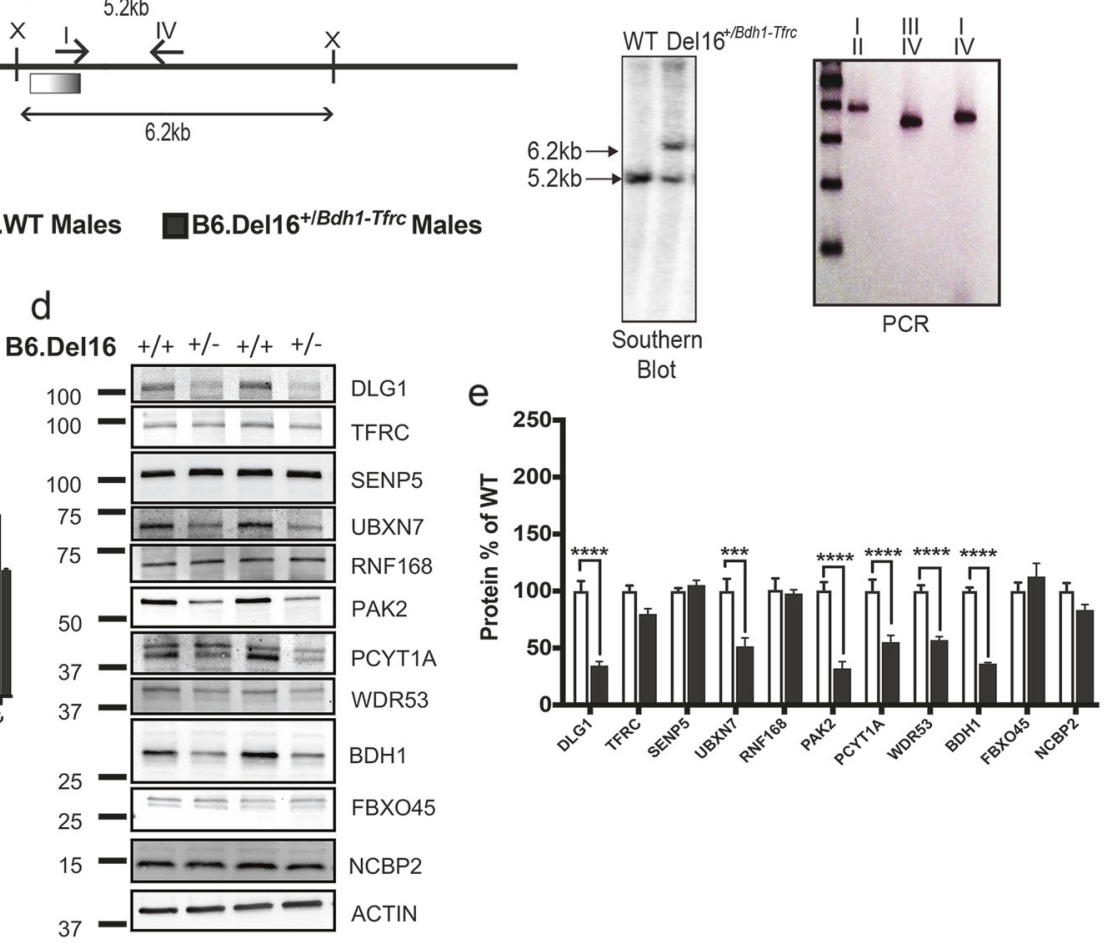

Fig. 1 Generation of $3 \mathrm{q} 29$ deletion on the C57BL/6N background using CRISPR/Cas9. a The 3q29 region is located on human chromosome 3 [recurrent deletion coordinates (GRCh38.p12)Chr3:195,998,129-197,623,129], and the syntenic 3q29 region is located on mouse chromosome 16 [mouse 3q29 deletion coordinates (GRCm38.p3)-Chr16:31,369,117-32,634,414]. b Two CRISPRs were designed (CRISPR-A [Chr.16:31,369,117] and CRISPR-B [Chr.16:32,634,414]) to create a heterozygous 3q29 deletion. The deletion was confirmed using southern blots $(5.2 \mathrm{~kb}=$ wild-type band, $6.2 \mathrm{~kb}=$ deletion band) and PCR. c B6.Del16 $6^{+/ B d h l-T f r c}$ male mice have

$\sim 50 \%$ decreased gene expression in most of the genes within the $3 q 29$ interval (with the exception of $T f r c$ ). All genes are significantly reduced $(p<0.0001)(N=4$ wild-type, 4 mutant; 3 technical replicates per sample). d, e Protein expression analyses reveals a significant reduction in DLG1, UBXN7, PAK2, PCYT1A, WDR53, and BDH1 $(* * * p<0.0005, * * * * p<0.0001)(N=6$ wild-type, 6 mutant ([for UBXN7 and RNF168 $N=4$ wild-type, 4 mutant]. All data analyzed by two-way, repeated-measures ANOVA followed by multiple comparisons with Sidak's correction. Results represent mean \pm SEM 
found that expression of 18/19 genes tested was 50\% reduced in female (Supplemental Fig. 1a) and male (Fig. 1c) B6.Del16 ${ }^{+/ B d h l-T f r c}$ mice compared with B6.WT littermates. The exception was $T f r c$, which was not significantly reduced in female B6.Del16 $6^{+/ B d h l-T f r c}$ mice, and only $30 \%$ reduced in male B6.Del16 ${ }^{+/ B d h l-T f r c}$ mice. We then examined protein expression of 11 of the highestexpressed genes by western blot. In both females (Supplemental Fig. 1b-c) and males (Fig. 1d, e), we found that only 6 of 11 proteins (DLG1, UBXN7, PAK2, PCYT1A, WDR53, and BDH1) were found to be significantly reduced in B6.Del16 $6^{+/ B d h l-T f r c}$ mouse forebrain tissue compared with B6.WT littermates; no genotype differences were detected for TFRC, SENP5, RNF168, FBXO45, and NCBP2. Thus, although the transcripts for 18 genes in the interval have decreased expression in a manner consistent with their haploinsufficiency, the corresponding protein levels are not always changed in B6. Del16 $6^{+/ B d h l-T f r c}$ mice. These data demonstrate that B6. Del16 ${ }^{+/ B d h 1-T f r c}$ mice recapitulate the genetic lesion in 3 q29 deletion syndrome and suggest leads for phenotypic driver genes.

\section{B6.Del16 ${ }^{+/ B d h 1-T f r c}$ display growth deficits}

Individuals with the 3q29 deletion demonstrate reduced weight at birth, and this may persist throughout childhood [2]. To assess whether the B6.Del16 ${ }^{+/ B d h l-\text { Tfrc }}$ mice display a growth phenotype, we generated cohorts of female and male
B6.Del16 ${ }^{+/ B d h 1-T f r c}$ mice along with wild-type (B6.WT) littermate controls. We weighed mice weekly over 16 weeks (starting at P8) and found that female B6.Del16 ${ }^{+/ B d h l-T f r c}$ mice weighed on average $2.24 \mathrm{~g}$ less than WT littermates $(p<0.0001)$ and male B6.Del16 ${ }^{+/ B d h l-T f r c}$ weighed on average $1.61 \mathrm{~g}$ less than WT littermates $(p<0.0005$, Fig. 2a, b). These data indicate that the B6.Del16 $6^{+B d h l-T f r c}$ mice recapitulate the reduced growth observed in study subjects with the $3 \mathrm{q} 29$ deletion.

We then assessed B6.Dlg $1^{+/}$mice for weight deficits over 16 weeks (starting at P8). Female B6.Dlg ${ }^{+/-}$mice weigh an estimated $0.78 \mathrm{~g}$ less than WT $(p<0.05)$. The weight effect was not seen in male B6.Dlg $1^{+/-}$mice $(p>0.05$, Fig. 2c, d). The effect sizes for the orthologous 3q29 deletion are significantly larger than the effect sizes for the $D \lg l$ heterozygote for females $(p<0.0005)$. Taken together, these data indicate that the growth phenotype in B6.Del16 $6^{+/ B d h l-T f r c}$ mice is not due to heterozygosity of $D \lg 1$ alone.

\section{B6.Del16 ${ }^{+/ B d h 1-T f r c}$ mice have smaller brains, but normal brain architecture}

We examined whole brains in B6.Del16 ${ }^{+/ B d h l-T f r c}$ mice for gross structural or anatomical changes. Both female $\left(t_{21}=\right.$ 7.316, $p<0.0001)$ and male $\left(t_{15}=5.231, p<0.0005\right)$ B6. Del16 ${ }^{+/ B d h 1-T f r c}$ brains are smaller compared with B6.WT littermates (Supplemental Fig. 2a). However, when we normalized the brain weight to body weight, we found that the brain:body weight ratio was increased in female
Fig. 2 Growth deficits in B6. Del16 ${ }^{+/ B d h l-T f r c}$ mice and female B6.D $\lg 1^{+/-}$mice. a Female $(* * * * p<0.0001)[N=$ 34 wild-type, 32 mutant] and b male $(* * * p<0.0005)[N=$ 33 wild-type, 27 mutant] B6. Del16 ${ }^{+/ B d h l-\text { Tfrc }}$ mice weigh significantly less by genotype compared with B6.WT littermates. c Female $\left({ }^{*} p<0.05\right)[N=23$ wild-type, 25 mutant], but not d male $(p>0.05)[N=36$ wild-type, 30 mutant], B6.Dlg $1^{+/-}$mice weigh significantly less compared with their B6.WT littermates. Weight data analyzed as outlined in methods
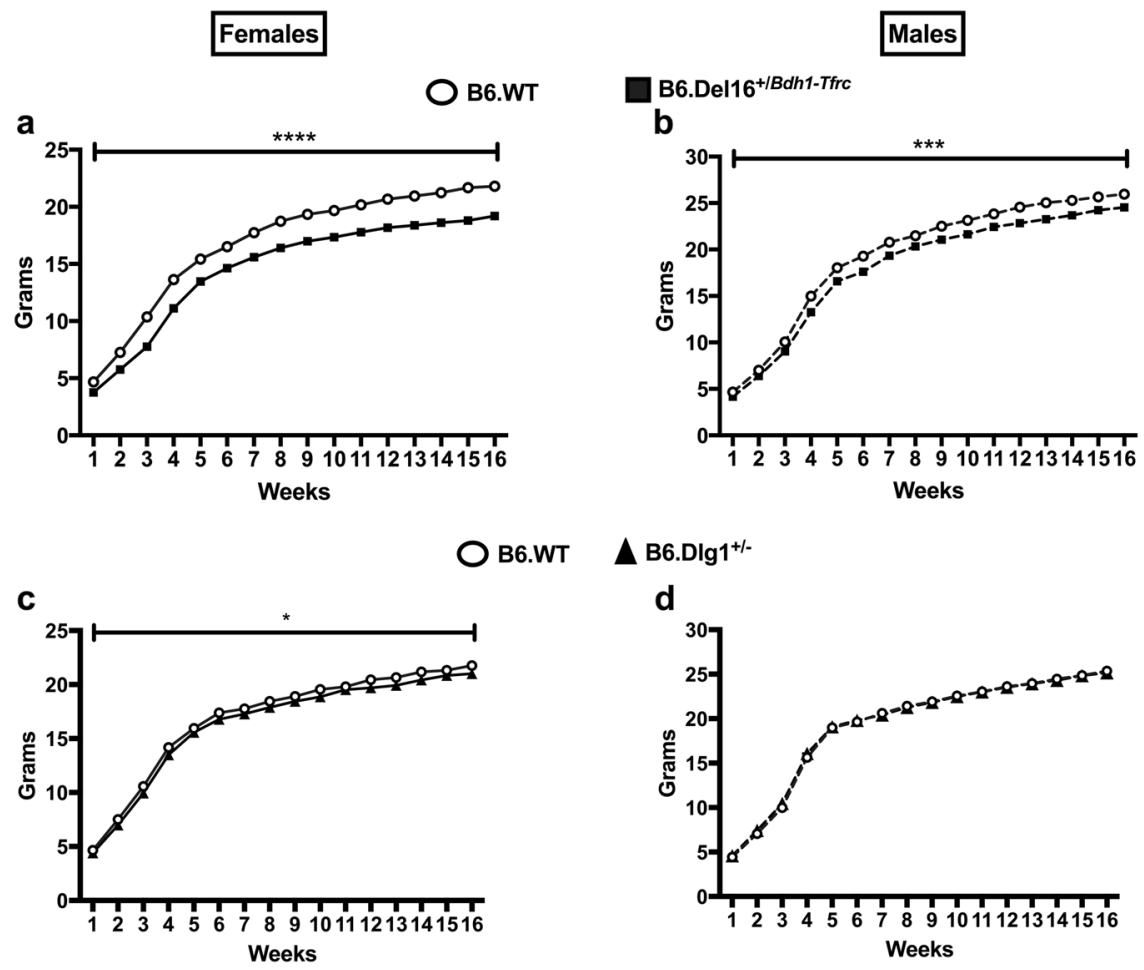

B6.WT

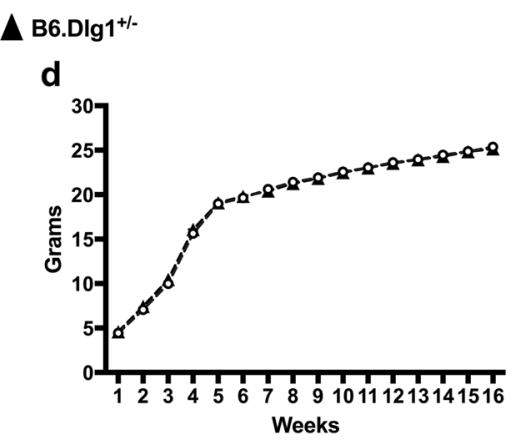


$\left(t_{21}=3.876, \quad p<0.001\right), \quad$ but not male $\left(t_{15}=0.4154\right.$, $p>0.05)$, B6.Del16 $6^{+/ B d h l-\text { Tfrc }}$ brains compared with B6.WT littermates (Supplemental Fig. 2b). We analyzed adult coronal sections with a cresyl violet stain and saw no gross differences in B6.Del16 ${ }^{+/ B d h l-T f r c}$ mice compared with their B6.WT littermates for either sex (Supplemental Fig. 2c, d). We assessed for any perturbation in the cortical plate using an antibody for T-box, Brain1 (TBR1). In E15.5 embryos, B6.Del16 $6^{+/ B d h l-T f r c}$ mice appeared to specify the cortical plate normally (Supplemental Fig. 3). Collectively, these data revealed altered brain size but no obvious architectural phenotypes in B6.Del16 ${ }^{+/ B d h 1-T f r c}$ brain morphology.

\section{B6.Del16 ${ }^{+/ B d h 1-\text { Ifrc }}$ and B6.DIg $1^{+/-}$have normal locomotion}

We assessed locomotion in B6.Del16 $6^{+/ B d h l-T f r c}$ and B6. $D \lg 1^{+/-}$mice using automated activity chambers by recording the number of ambulations within the chambers. We found no differences in ambulations immediately following exposure to a novel environment or over the ensuing $24 \mathrm{~h}$ in either female (main effect of genotype, $F_{1,30}=2.387, p>0.05$ ) or male (main effect of genotype, $\left.F_{1,28}=0.3563, p>0.05\right)$ B6.Del16 ${ }^{+/ B d h l-T f r c}$ mice (Supplemental Fig. 4a, b). Similarly, B6.Dlg ${ }^{+/-}$females (main effect of genotype, $F_{1,15}=0.3667, p>0.05$ ) or males (main effect of genotype, $F_{1,11}=0.3087, p>0.05$ ) (Supplemental Figs. 4c, d) also showed no differences in ambulations compared with their respective B6.WT littermates. Thus, neither B6.Del16 $6^{+/ B d h 1-T f r c}$ nor B6.D $\lg 1^{+/}$ mice have locomotor deficits, suggesting that behavioral phenotypes can be assessed, as the mice do not exhibit an obvious movement confound.

\section{B6.Del16 ${ }^{+/ B d h 1-T f r c}$ male mice have social interaction deficits}

Because ASD is one of the phenotypes reported in $3 \mathrm{q} 29$ deletion patients [2, 27], we tested the mice for social interaction deficits using the three-chamber social approach task. We measured duration of olfactory investigation to gauge how much a given subject mouse was interacting either with the empty cup or with the cup containing the stranger mouse. As expected, both female $\left(t_{26}=7.176, p<0.0001\right)$ and male $\left(t_{28}=4.018, p<0.0005\right) \mathrm{B} 6$.WT interacted significantly more with the stranger mouse compared with the empty cup. Female $\left(t_{24}=3.237, \quad p<0.005\right) \quad$ B6.Del16 ${ }^{+/ B d h l-T f r c}$ also showed normal sociality. By contrast, male $\left(t_{28}=1.769, p=\right.$ 0.0878) B6.Del16 ${ }^{+/ B d h l-T f r}$ mice exhibited abnormal sociality as they did not show a significant preference for the stranger mouse over the empty cup (Fig. 3a). We then tested female and male B6.Dlg1 $1^{+-}$mice to determine whether haploinsufficiency of $\mathrm{Dlg} 1$ led to social impairment (Fig. 3b). We did not observe any deficits in either female $\left(t_{24}=4.716, p<\right.$ $0.0001)$ or male $\left(t_{24}=2.846, p<0.01\right) \mathrm{B} 6 . D \lg 1^{+/-}$mice or their B6.WT littermates [female $\left(t_{26}=3.711, p<0.005\right)$, (male $\left.\left.t_{22}=6.29, p<0.0001\right)\right]$. These data indicate that male B6.Del16 $6^{+/ B d h l-T f r}$ mice display social impairment that is not solely attributable to $D l g l$ haploinsufficiency.

\section{B6.Del16 ${ }^{+/ B d h 1-T f r c}$ male mice have spatial memory deficits}

In the MWM, female B6.Del16 $6^{+/ B d h l-T f r c}$ mice showed a similar pattern of learning compared with their B6.WT littermates as there was no difference in latency (main effect of genotype, $F_{1,30}=0.07352, p>0.05$ ), or distance (main effect of genotype, $F_{1,30}=0.6494, p>0.05$ ) to find the hidden platform during the training portion of the MWM. Although there was no genotype difference in swim speed (main effect of genotype, $F_{1,30}=2.188, p>0.05$ ), there was a significant interaction (genotype $\times$ time, $F_{4,120}, p<0.005$ ). Sidak's posthoc analysis revealed female B6.Del16 ${ }^{+/ B d h l-\text { Tfrc }}$ mice swim faster on day 5 compared with B6.WT littermates $(p<0.005)$ (Supplemental Fig. 5a-c). Male B6.Del16 $6^{+ \text {Bdhl-Tfrc mice }}$ showed a trend towards increased latency that did not reach significance (main effect of genotype, $F_{1,28}=3.922, p=$ 0.057), swam a greater distance (main effect of genotype, $F_{1,28}=4.621, p<0.05$ ), but had similar swim speed (main effect of genotype, $F_{1,28}=0.493, p>0.05$ ) to reach the hidden platform on day 5 compared with B6.WT littermates (Supplemental Fig. 5d-f). We then tested for spatial memory deficits in the probe trial (Fig. 3c) and found no difference in percentage of time spent in the quadrant that formerly contained the platform between female B6. Del16 ${ }^{+/ B d h l-\text { Tfrc }}$ mice and their B6.WT littermates $\left(t_{30}=\right.$ 0.8357, $p>0.05)$. However, male B6.Del16 $6^{+/ B d h l-T f r c}$ mice spent significantly less time in the platform quadrant compared with their B6.WT littermates $\left(t_{28}=2.592, p<0.05\right)$.

We tested both female and male B6.Dlg ${ }^{+/-}$mice in the MWM. There were no significant differences in latency (main effect of genotype, $F_{1,17}=0.1776, p>0.05$ ), distance (main effect of genotype, $F_{1,17}=0.1407, p>0.05$ ), or swim speed (main effect of genotype, $F_{1,17}=0.5809, p>0.05$ ) in female (Supplemental Fig. 6a-c) or male [latency: (main effect of genotype, $\left.F_{1,16}=0.2816, p>0.05\right)$, distance: (main effect of genotype, $F_{1,16}=0.3065, p>0.05$ ), speed: (main effect of genotype, $F_{1,16}=0.0031, p>0.05$ ) (Supplemental Fig. 6d-f) B6.Dlg1 ${ }^{+/}$mice compared with B6. WT littermates. During the probe trial (Fig. 3d), both female $\left(t_{17}=0.5685, p>0.05\right)$ and male $\left(t_{16}=1.573, p>0.05\right) \mathrm{B} 6$. $D \lg 1^{+/-}$mice spent a similar amount of time in the platform quadrant compared with B6.WT littermates. Collectively, these data show male B6.Del16 ${ }^{+/ B d h l-T f r c}$ mice exhibit spatial learning and memory deficits that are not solely due to haploinsufficiency of $\mathrm{Dlgl}$. 


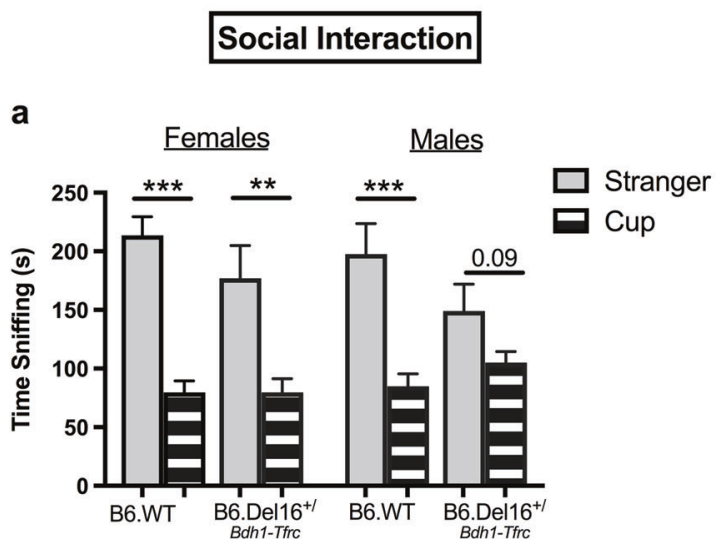

b

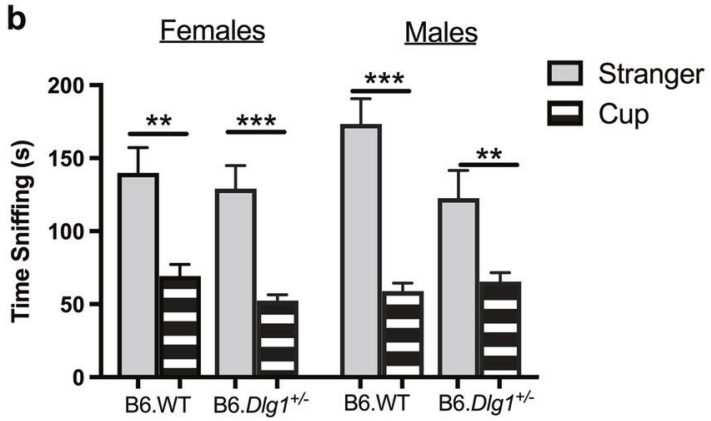

Fig. 3 Social and cognitive impairment in male B6.Del16 ${ }^{+/ B d h l-T f r c}$ mice. a Female B6.Del16 $6^{+/ B d h l-T f r c}$ mice interact significantly more with the stranger under the cup compared with empty cup alone $(* * p<0.01)$ similar to their B6.WT littermates $(* * * p<0.0005)[N=$ 14 wild-type, 14 mutant]. Male B6.Del16 ${ }^{+/ B d h 1-\text { Tfrc }}$ mice do not show a significant preference for interacting with either the stranger under the cup or the empty cup $(p=0.09)$. Male B6.WT mice significantly prefer to interact with the stranger under the cup compared with the empty cup $(* * * p<0.0005)[N=15$ wild-type, 15 mutant]. b Female B6.Dlg $1^{+/-}$mice interact significantly more with the stranger under the cup compared with empty cup alone $(* * * p<0.0005)$ ) similar to their B6.WT littermates $(* * p<0.01))[N=14$ wild-type, 13 mutant]. Male B6.Dlg $1^{+/-}$mice interact significantly more with the

\section{B6.Del16 ${ }^{+/ B d h 1-T f r c}$ mice display increased startle response}

We examined potential differences in response to an acoustic startle and in sensorimotor gating using PPI. Female B6.Del16 ${ }^{+/ B d h l-T f r c}$ mice (Fig. 4a) displayed a significant increase in acoustic startle response compared with their B6.WT littermates $(p<0.05)$. Male B6.Del16 $6^{+/ B d h l-\text { Tfr }}$ mice showed a similar trend of increased startle, but it did not reach significance $(p=0.056)$ (Fig. $4 \mathrm{~b})$. Because the magnitude of the measured startle response can be affected by weight, we performed statistical analyses to correct for the decreased weight of B6.Del16 $6^{+/ B d h l-\text { Tfrc }}$ mice. Our analyses revealed that weight did not contribute significantly to startle response $(p>0.25)$, indicating that the weight deficits observed in B6.Del16 $6^{+/ B d h l-T f r c}$ mice did not affect the
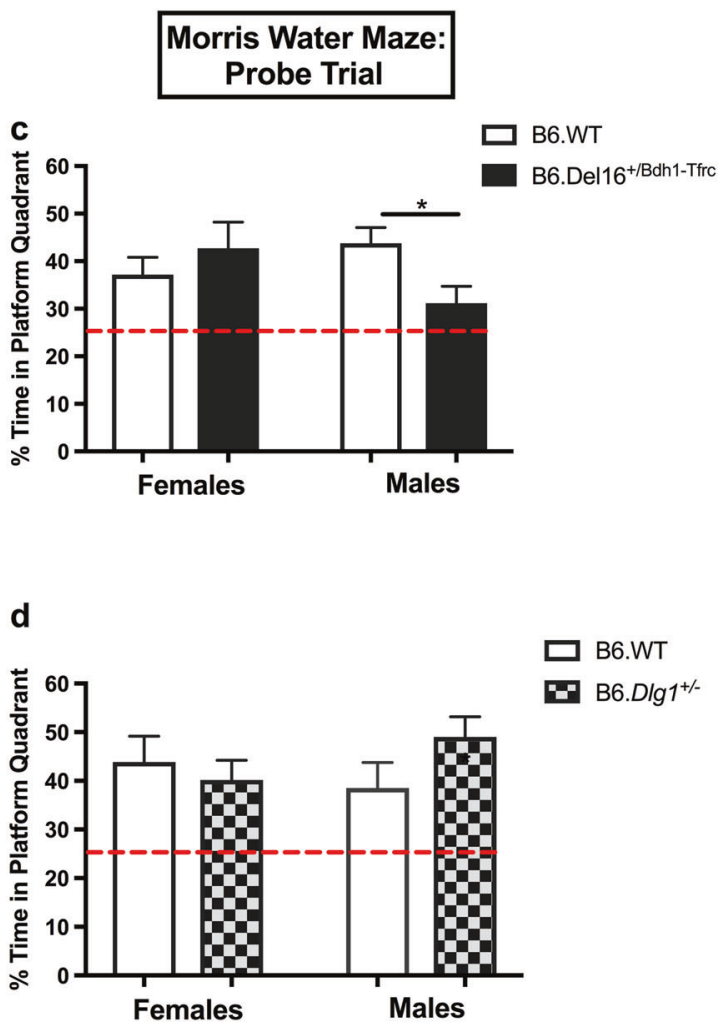

stranger under the cup compared with the empty cup $(* * p<0.01)$ similar to their B6.WT littermates $(* * * p<0.0005)[N=12$ wild-type, 13 mutant]. c Female B6.Del16 ${ }^{+/ B d h l-\text { Tfrc }}$ mice spend a similar amount of time in the platform quadrant compared with B6.WT littermates $(p>0.05)\left[N=16\right.$ wild-type, 16 mutant]. Male B6.Del16 ${ }^{+/ B d h l-\text { Tfrc }}$ mice spend significantly less time in the platform quadrant compared with B6.WT littermates $(* p<0.05)[N=15$ wild-type, 15 mutant $]$. d Female $(p>0.05)$ [8 wild-type, 11 mutant] and male $(p>0.05)[9$ wild-type, 9 mutant] B6.Dlg $1^{+-}$mice spend a similar time in the platform quadrant compared with their B6.WT littermates. The dashed line denotes $25 \% / c h a n c e$. All data analyzed by two-tailed Student's $t$ test. Results represent the mean \pm SEM

measured startle response. Female (Fig. 4c) (main effect of genotype, $F_{1,17}=0.6714, p>0.05$ ) and male (Fig. $4 \mathrm{~d}$ ) (main effect of genotype, $\left.F_{1,16}=0.4238, p>0.05\right)$ B6. $\lg 1^{+/}$mice displayed normal acoustic startle. We assessed for deficits in sensorimotor gating using PPI. Female B6.Del16 $6^{+/ B d h l-T f r c}$ mice had similar PPI compared with B6.WT littermates (main effect of genotype, $F_{1,30}=0.083$ ), but did have a significant interaction (genotype $\times$ prepulse, $F_{3,90}=3.527$, $p<0.05)$. Sidak's post-hoc analysis revealed no significant differences at the respective prepulses $(p>0.05)$. Male B6. Del16 ${ }^{+/ B d h l-T f r c}$ mice did not show any differences compared with B6.WT littermates (main effect of genotype, $\left.F_{1,28}=0.1715, \quad p>0.05\right)$. Furthermore, neither female (main effect of genotype, $F_{1,17}=1.654, p>0.05$ ) nor male (main effect of genotype, $F_{1,16}=1.998, p>0.05$ ) B6.Dlg $1^{+/}$ mice showed any differences in PPI compared with their 


\section{O/口B6.WT \\ $\square / \square$ B6.Del16 ${ }^{+/ B d h 1-T f r C}$}

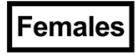

a
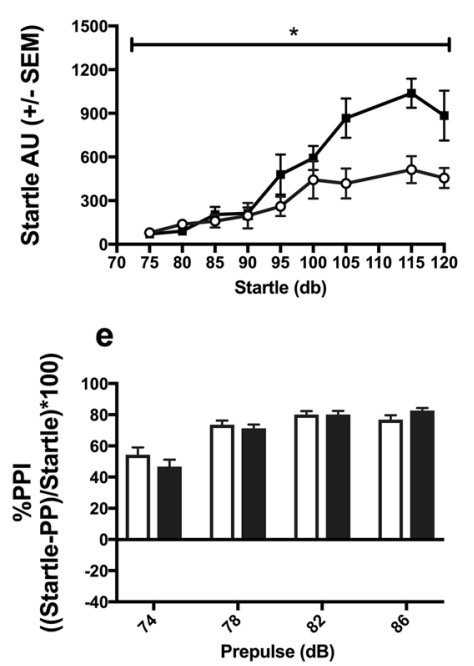

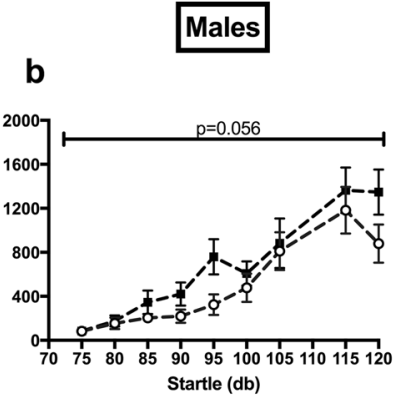

f

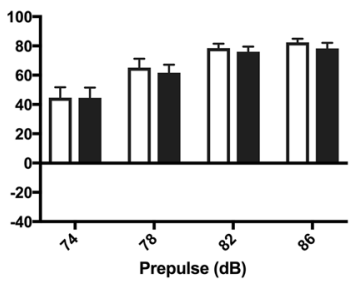

O/ロ B6.WT

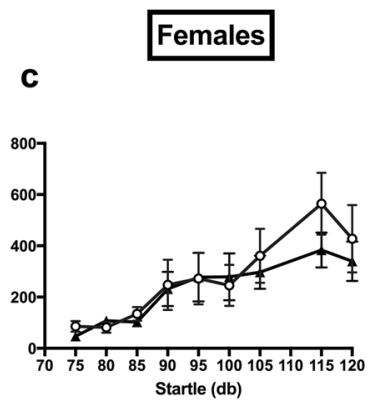

g

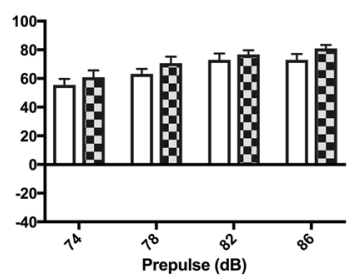

$\Delta / E B 6 . D \lg 1^{+/-}$

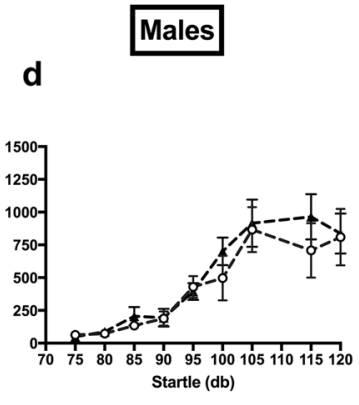

h

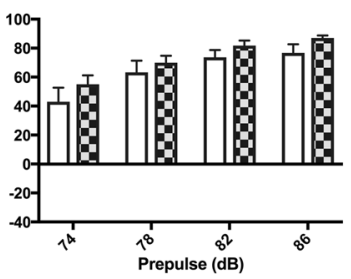

Fig. 4 Increased startle, but normal prepulse inhibition, in B6.Del16 ${ }^{+/ B d h l-T f r c}$ mice. a Female $[N=16$ wild-type, 16 mutant $]$ and b male $[N=15$ wild-type, 15 mutant $]$ B6.Del16 $6^{+/ B d h l-T f r c}$ mice startle more compared with B6.WT littermates. c Female $[N=8$ wild-type, 11 mutant $]$ and $\mathbf{d}$ male $[N=9$ wild-type, 9 mutant $]$ B6.Dlg $1^{+/}$mice startle similarly to B6.WT littermates. e Female $[N=16$ wild-type, 16 mutant]; f male [ $N=15$ wild-type, 15 mutant] B6.Del16 ${ }^{+/ B d h l-\text { Tfrc }}$ mice

B6.WT littermates (Figs. 4e-h). These data indicate that mice harboring the 3q29 deletion have an increased response to an acoustic startle but mostly normal sensorimotor gating, whereas happloinsufficiency of $D l g 1$ does not alter either measure.

\section{Amphetamine-induced locomotor activity is attenuated in B6.Del16 ${ }^{+/ B d h 1-\text { Ifrc }}$ mice}

Chronic amphetamine use can lead to SZ symptoms, and amphetamine can exacerbate SZ symptoms in individuals that already have the disorder [28]. Because of the strong association between the 3q29 deletion and SZ, we tested amphetamine sensitivity in both B6.Del16 ${ }^{+/ B d h l-T f r c}$ and B6. $D \lg 1^{+/}$mice. Following saline administration, neither female nor male B6.Del16 ${ }^{+/ B d h l-T f r c}$ mice displayed significant differences in ambulatory activity compared with their B6.WT littermates (main effect of genotype, $p>0.05$ ). At both the 2.5 and $7.5 \mathrm{mg} / \mathrm{kg}$ doses of amphetamine, both female and male B6.WT mice were significantly more active compared with the respective B6.WT mice that received saline (main effect of treatment, $p<0.0001)$. Amphetamine-induced locomotion was attenuated in female B6.Del16 ${ }^{+/ B d h l-T f r c}$ mice following the $2.5 \mathrm{mg} / \mathrm{kg}$ (treatment $\times$ genotype interaction, $p<0.005$ ) and $7.5 \mathrm{mg} / \mathrm{kg}$ (treatment $\times$ genotype interaction, $p<0.0001$ ) doses compared with B6.WT littermates (Fig. 5a). Male B6. have similar prepulse inhibition compared with B6.WT littermates. g Female $[N=8$ wild-type, 11 mutant $]$ and $\mathbf{h}$ male $[N=9$ wild-type, 9 mutant] B6.Dlg $1^{+/}$mice have similar prepulse inhibition compared with B6.WT littermates. Startle data analyzed as outlined in methods. PPI data analyzed by two-way, repeated-measures ANOVA followed by multiple comparisons. Results represent the mean \pm SEM $(* p<$ $0.05)$

Del16 ${ }^{+/ B d h l-T f r c}$ mice did not show any differences in activity following the $2.5 \mathrm{mg} / \mathrm{kg}$ dose (treatment $\times$ genotype interaction, $p>0.05$ ), but did show a reduction in activity following the $7.5 \mathrm{mg} / \mathrm{kg}$ dose (treatment $\times$ genotype interaction, $p<$ 0.0001) of amphetamine compared with B6.WT littermates (Fig. 5b). Because stereotypy can occlude horizontal locomotion at high doses of amphetamine, we video recorded mice from the 35-65 min post-injection and manually scored the videos for stereotypy as previously reported [23], but did not observe any significant differences (data not shown). We then assessed amphetamine sensitivity in B6.DlgI ${ }^{+/}$mice. Neither female nor male B6.Dlg $I^{+/}$mice displayed significant differences in ambulatory activity compared with their B6.WT littermates following administration of saline (main effect of genotype, $p>0.05$ ). At both the 2.5 and 7.5 $\mathrm{mg} / \mathrm{kg}$ dose of amphetamine, both female and male B6.WT mice were significantly more active compared with the respective B6.WT mice that received saline (main effect of treatment, $p<0.0001$ ). Female B6.Dlg $1^{+/}$mice did not display differences in activity at the $2.5 \mathrm{mg} / \mathrm{kg}$ (treatment $\times$ genotype interaction, $p>0.05$ ) or $7.5 \mathrm{mg} / \mathrm{kg}$ (treatment $\times$ genotype interaction, $p>0.05$ ) amphetamine doses compared with their B6.WT littermates (Fig. 5c). Male B6.Dlg ${ }^{+/-}$mice showed a small but significant increase in ambulatory activity following administration of $2.5 \mathrm{mg} / \mathrm{kg}$ amphetamine (treatment $\times$ genotype interaction, $p<0.001$ ), but no significant 


\section{Females}

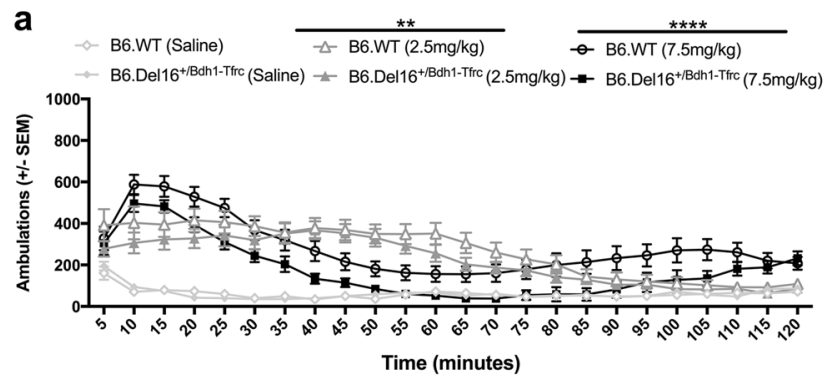

C

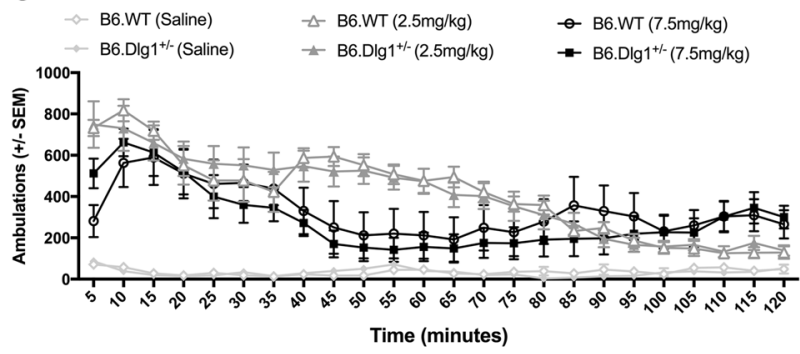

Fig. 5 Attenuated activity after administration of amphetamine in B6. Del16 ${ }^{+/ B d h l-T f r c}$ mice. a Female B6.Del16 $6^{+/ B d h l-\text { Tfrc }}$ mice displayed no significant differences in ambulatory activity compared with B6.WT littermates after saline administration (main effect of genotype, $p>$ $0.05)$. Female B6.WT mice displayed increased activity when given a $2.5 \mathrm{mg} / \mathrm{kg}$ or a $7.5 \mathrm{mg} / \mathrm{kg}$ dose of amphetamine compared with B6.WT littermates that received saline (main effect of treatment, $p<0.0001$ ). Female B6.Del16 ${ }^{+/ B d h l-T f r c}$ mice displayed attenuated activity following a $2.5 \mathrm{mg} / \mathrm{kg}$ dose (treatment $\times$ genotype interaction, ${ }^{* *} p<0.005$ ) and a $7.5 \mathrm{mg} / \mathrm{kg}$ dose (treatment $\times$ genotype interaction, $* * * * p<$ 0.0001) of amphetamine compared with B6.WT littermates. b Male B6.Del16 ${ }^{+/ B d h I-\text { Tfr }}$ mice displayed no significant differences in ambulatory activity compared with B6.WT littermates after saline administration (main effect of genotype, $p>0.05$ ). Male B6.WT mice displayed increased activity when given a $2.5 \mathrm{mg} / \mathrm{kg}$ or a $7.5 \mathrm{mg} / \mathrm{kg}$ dose of amphetamine compared with B6.WT littermates that received saline (main effect of treatment, $p<0.0001$ ). Male B6.Del16 ${ }^{+/ B d h I-T f r c}$ mice displayed no significant changes in activity following a $2.5 \mathrm{mg} / \mathrm{kg}$ dose (treatment $\times$ genotype interaction, $p>0.05$ ), but did show attenuated activity following a $7.5 \mathrm{mg} / \mathrm{kg}$ dose (treatment $\times$ genotype interaction, $* * * * p<0.0001)$ of amphetamine compared with B6.WT littermates. c Female B6.Dlg $1^{+/}$mice displayed no significant

changes in activity following the $7.5 \mathrm{mg} / \mathrm{kg}$ dose (treatment $\times$ genotype interaction, $p>0.05$ ) compared with B6.WT littermates (Fig. 5d). Taken together, these data show that B6. Del16 ${ }^{+/ B d h l-T f r c}$ mice display attenuated amphetamine-induced locomotor activity that is not solely due to haploinsufficiency of $\mathrm{Dlgl}$.

\section{Anxiety-like behavior and associative memory are not changed in B6.Del16 ${ }^{+/ B d h 1-\text { ffrc }}$ and B6.DIg $1^{+/-}$}

We assessed performance in several tests for anxiety-like behavior (elevated plus maze, open field, marble burying) and did not observe any differences in the respective tasks
Males

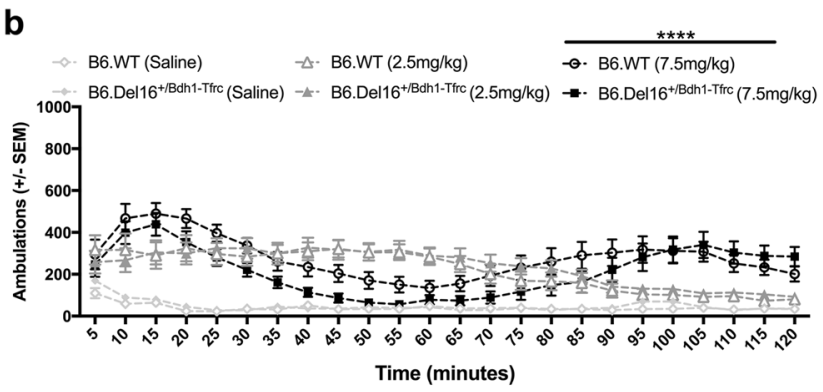

d

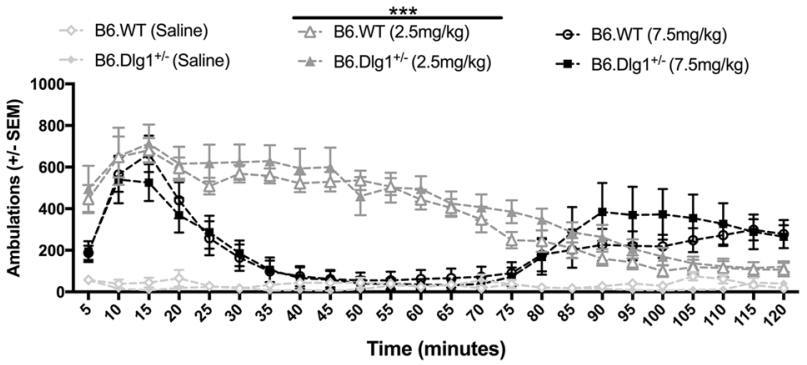

differences in ambulatory activity compared with B6.WT littermates after saline administration (main effect of genotype, $p>0.05$ ). Female B6.WT mice displayed increased activity when given a $2.5 \mathrm{mg} / \mathrm{kg}$ or a $7.5 \mathrm{mg} / \mathrm{kg}$ dose of amphetamine compared with B6.WT littermates that received saline (main effect of treatment, $p<0.0001$ ). Female B6. $D \lg 1^{+/}$mice displayed no changes in activity after a $2.5 \mathrm{mg} / \mathrm{kg}$ dose (treatment $\times$ genotype interaction, $p>0.05$ ) or a $7.5 \mathrm{mg} / \mathrm{kg}$ dose (treatment $\times$ genotype interaction, $p>0.05$ ) compared with B6.WT littermates. d Male B6.Dlg $1^{+/-}$mice displayed no significant differences in ambulatory activity compared with B6.WT littermates after saline administration (main effect of genotype, $p>0.05$ ). Male B6.WT mice displayed increased activity when given a $2.5 \mathrm{mg} / \mathrm{kg}$ or a $7.5 \mathrm{mg} / \mathrm{kg}$ dose of amphetamine compared with B6.WT littermates that received saline (main effect of treatment, $p<0.0001$ ). Male B6.Dlg $1^{+/-}$mice displayed increased activity following a $2.5 \mathrm{mg} / \mathrm{kg}$ dose (treatment $\times$ genotype interaction, ${ }^{* * *} p<0.001$ ), but not a $7.5 \mathrm{mg} / \mathrm{kg}$ dose (treatment $\times$ genotype interaction, $p>0.05$ ) of amphetamine compared with B6.WT littermates. All data were analyzed as outlined in methods. Results represent mean \pm SEM $(* p<0.05)$. B6.Del16 ${ }^{+/ B d h l-T f r c}$ micefemales: $N=16$ wild-type, 16 mutant; males: $N=14$ wild-type, 15 mutant. B6.Dlg1 $1^{+-}$mice-females: $N=8$ wild-type, 10-11 mutant; males: 8-9 wild-type, 9 mutant

$(p>0.05)$ in B6.Del16 $6^{+/ B d h l-T f r c}$ or B6.Dlg1 $1^{+/-}$mice compared with B6.WT littermates (Supplemental Fig. 7) [statistics located in respective figure legend].

We assessed associative learning and memory using context and cued fear conditioning. During training, female B6. Del16 $6^{+/ B d h l-T f r c}$ mice did not show any genotype-wide differences compared with B6.WT littermates, but did show a significant interaction (main effect of time $\times$ genotype, $F_{21,630}$, $p<0.0005$ ). Sidak's post-hoc analysis revealed female B6. Del16 ${ }^{+/ B d h l-T f r c}$ mice froze significantly more at the $300(p<$ $0.01), 360(p<0.05)$, and $380(p<0.0001)$ second time points compared with B6.WT littermates (Supplemental Fig. 8a). This result is consistent with the increased acoustic startle 
phenotype and suggests that female B6.Del16 $6^{+B d h l-T f r c}$ mice are more responsive to the immediate physiological and/or psychological effects of aversive stimuli. During the context (main effect of genotype, $F_{1,30}=0.1885, p>0.05$ ) and cue (main effect of genotype, $F_{1,30}=2.682, p>0.05$ ) tests, female B6.Del16 ${ }^{+/ B d h l-T f r c}$ mice had similar freezing percentages compared with B6.WT littermates (Supplemental Fig. 8b, c). Male B6.Del16 ${ }^{+/ B d h l-T f r c}$ mice did not show any differences during the training (main effect of genotype, $F_{1,28}=0.248$, $p>0.05$ ), context (main effect of genotype, $F_{1,28}=0.9298$, $p>0.05$ ), or cue (main effect of genotype, $F_{1,28}=1.981, p>$ 0.05 ) phases compared with B6.WT littermates during any of the phases (Supplemental Fig. 8d-f).

During training, female B6.Dlg1 $1^{+/}$mice froze significantly less (main effect of genotype, $F_{1,17}=5.096, p<$ 0.05 ; main effect of genotype $\times$ time, $F_{21,357}=2.006, p<$ 0.01) compared with B6.WT littermates (Supplemental Fig. 9a). Sidak's post-hoc analysis revealed B6.Dlg $1^{+/}$ females froze significantly less at $320 \mathrm{~s}(p<0.01)$ and $380 \mathrm{~s}$ $(p<0.001)$. In the context test, female B6.Dlgl ${ }^{+/-}$had similar freezing percentages compared with B6.WT littermates (main effect of genotype, $F_{1,17}=1.703, p>0.05$ ) (Supplemental Fig. 9b), whereas female B6.Dlg $1^{+/-}$mice froze less (main effect of genotype, $F_{1,17}=4.302, p=0.05$ ) during the cue test (Supplemental Fig. 9c). During training (main effect of genotype, $F_{1,16}=0.291, p>0.05$ ), context (main effect of genotype, $F_{1,16}=0.4616, p>0.05$ ) and cue tests (main effect of genotype, $F_{1,16}=0.007, p>0.05$ ), male B6.Dlg $1^{+/-}$mice have a similar freezing percentage compared with B6.WT littermates (Supplemental Fig. 9e, f). Collectively, we did not observe fear-dependent memory impairments in B6.Del16 ${ }^{+/ B d h l-T f r c}$ mice.

\section{Discussion}

Here we report engineering a 3q29 deletion mouse model, and show it displays growth and behavioral deficits relevant to human 3q29 deletion syndrome phenotypes. Both female and male B6.Del16 $6^{+/ B d h l-T f r c}$ mice weigh significantly less than their B6.WT littermates, over the course of postnatal development and into adulthood, consistent with a prior study of humans with 3q29 deletion syndrome [2]. In contrast, female but not male B6.Dlg1 $1^{+/}$mice weigh significantly less than their B6.WT littermates suggesting that haploinsufficiency of $D \lg 1$ may be a partial contributor to the growth phenotype in female B6.Del16 ${ }^{+/ B d h l-T f r c}$ mice. We found multiple behavioral differences in B6. Del16 ${ }^{+/ B d h l-T f r c}$ mice that are endophenotypes of the neuropsychiatric disorders observed in 3q29 deletion study subjects $[2,9,12]$. Male B6.Del16 ${ }^{+/ B d h 1-T f r c}$ mice demonstrated social and cognitive impairment in the threechambered social interaction and MWM tests, respectively, consistent with ASD and intellectual disability seen in humans with 3q29 deletion syndrome. Female B6. Del16 ${ }^{+/ B d h l-T f r c}$ mice showed an increased startle response consistent with reported hypersensitivity to acoustic stimuli in ASD patients [29]. Female and male B6.Del16 ${ }^{+/ B d h l-T f r c}$ mice showed attenuated sensitivity to amphetamine-induced locomotor activity at a high dose of drug, suggesting perturbations in the dopaminergic system and demonstrating a shared behavior phenotype. Disruptions in dopamine signaling have been shown to confer risk for both ASD and SZ $[30,31]$. We also demonstrated that B6.Dlg ${ }^{+/-}$mice did not share any of the behavioral phenotypes that are observed in B6.Del16 $6^{+B d h l-T f r c}$ mice. These data indicate that B6. Del16 ${ }^{+/ B d h l-T f r c}$ mice display 3q29-related phenotypes that are not due solely to haploinsufficiency of $D \lg 1$.

The finding that the B6.Del16 ${ }^{+/ B d h l-T f r c}$ mouse recapitulates aspects of 3q29 deletion at the genetic and phenotypic levels strongly suggests that the genetic drivers are within the 3q29 interval. The major difference between the mouse and human intervals is that the syntenic regions are inverted. This inversion occurred within the great ape lineage, as chimpanzees and humans have the same (derived) orientation of the region, but rhesus macaques have the inverted (ancestral) orientation that is present in mice. A copy number neutral inversion of the region has been identified in humans, with no apparent phenotype [32]. This implies that the critical event for the phenotype is the deletion itself, not a misregulation of a gene outside the deletion region. The fact that all 21 genes in the human 3q29 region are conserved in mouse and single gene mutations of each are being analyzed on the same genetic background through the International Mouse Phenotyping Consortium argues the region is ripe for genetic dissection. The B6.Del16 $6^{+B d h l-T f r c}$ mouse provides an entry point for such genetic dissection and ultimately, understanding of the circuitry and molecular mechanisms underlying these phenotypes. Towards this goal, the behavioral deficits provide clues to which brain regions may be most affected by the 3q29 deletion: the MWM is a hippocampal-dependent task [33, 34], social interaction is a striatum-dependent task [35], and startle response is dependent on the caudal pontine reticular nucleus [36]. Thus, the observed deficits in B6. Del16 ${ }^{+/ B d h l-T f r c}$ mice that we observed in the MWM are potential disruptions in these respective brain regions. Such relationships provide a starting point for evaluating the circuitry that may be disrupted in the B6.Del16 $6^{+/ B d h l-\text { Tfrc }}$ mice, which may also be relevant for human 3q29 deletion patients.

While this current work focused on specific symptoms exhibited by $3 \mathrm{q} 29$ deletion patients, the mice have the potential to inform other 3q29 deletion phenotypes. The ongoing Emory 3q29 Project (http://genome.emory.edu/3q29/) has an active component where human study subjects participate in a comprehensive set of clinical evaluations [37]. Phenotypic data 
from the B6.Del16 $6^{+/ B d h l-T f r c}$ mice can inform human phenotyping protocols and downstream analyses. For example, we observed sex-specific differences in our behavioral assertation of the B6.Del16 $6^{+B d h l-T f r c}$ mice but only limited patient phenotypes have been evaluated in a sex-specific way. We suspect that the sex-based differences we observed in the mice are due to intrinsic biological differences between females and males. Certainly, sex-specific differences are exhibited in other neuropsychiatric diseases, notably ASD where four times as many males as females are affected [38-40]. Though purely speculative in relation to the $3 \mathrm{q} 29$ deletion, our behavior data demonstrates differences between B6.Del16 ${ }^{+/ B d h l-T f r c}$ females and male mice, which could be due to sex-specific molecular phenotypes. Although the formal possibility exists that the variability in estrous stage contributed to the sex differences we observed, we think this is an unlikely explanation. The females were group-housed, minimizing any such variation. Furthermore, male mice display as much variability in behavioral assays as female mice without estrous control [41]. These sexspecific differences are significant as they underscore the importance of studying both sexes in mice and humans.

Our $D \lg 1^{+/}$analyses argue that haploinsufficiency of Dlgl alone is not sufficient to explain the phenotypes associated with the B6.Del16 $6^{+/ B d h l-T f r c}$ mice, and points to either a different gene or a combination of genes within the interval that may or may not include $D \lg 1$ driving phenotypes. These data highlight the power of our approach. Recently, a neuron-specific deletion of $D l g l$ was reported to produce cognitive deficits in the males and motor learning deficits in the females [17]. While this work identified differentially expressed genes in the hippocampus, the transcriptional profile is likely much different when only one copy of $D L G 1$ is deleted as in 3q29 deletion patients. The tissue-specific complete deletion of $D \lg 1$ also focuses on the role in neurons, but other cell types, such as glia, may contribute to 3q29 deletion associated phenotypes.

One potential modifier of DLG1 is PAK2. In Drosophila, single pak- $/+$ or $d l g-/+$ mutants showed no phenotype but compound pak-I+; $d l g-I+$ flies showed decreased number of neuromuscular boutons indicating a genetic interaction [19]. Although this interaction remains unexamined in mouse, male $\mathrm{Pak}^{+/-}$mice are impaired on several social tasks including the three-chamber social interaction protocol used in the present study [18]. Diminished Pak2 likely contributes to the social impairment we observed in the B6. Del16 $6^{+/ B d h l-T f r c}$ male mice.

Several behavioral assays performed in the present study did not reveal phenotypes in the B6.Del16 $6^{+/ B d h l-\text { Tfr }}$ mice. Based on the known manifestations of 3q29 deletion in humans, we hypothesized that some of these phenotypes would be apparent, such as anxiety [2]. It is possible that more complex human life experiences such as early life stress contribute to these phenotypes. Alternatively, our mouse studies were conducted on the C57BL/6N inbred strain, which may have obscured modifying genetic elements. Thus, examining the Del16 $6^{+/ B d h l-\text { Tfrc }}$ on different genetic backgrounds could reveal additional impairments. It is also entirely possible that the distinctions in phenotype reflect fundamentally different biology between mice and humans. Nonetheless, the B6.Del16 $6^{+/ B d h l-T f r c}$ mouse will be an important tool to move forward and explore these issues and to systematically generate sub-deletions to genetically dissect the regions of the $3 q 29$ interval driving the observed phenotypes. This $3 q 29$ deletion mouse provides an excellent tool to continue the quest of unraveling the puzzle of neuropsychiatric disorders.

Acknowledgements The work was supported by R01GM097331 (TC, DW, and STW), R56MH116994 (TC, JGM, DW, and STW) and R01MH110701 along with funds from the Department of Human Genetics at Emory. This study was supported in part by the Mouse Transgenic and Gene Targeting Core (TMF) and the Rodent Behavioral Core, which are subsidized by the Emory University School of Medicine and are part of the Emory Integrated Core Facilities. Additional support was provided by the Georgia Clinical and Translational Science Alliance of the National Institutes of Health under Award Number UL1TR002378. The content is solely the responsibility of the authors and does not necessarily reflect the official views of the National Institutes of Health. We are grateful to Dr. Jeffrey Miner, Washington University in St Louis, for providing Dlg1 mutant mice. This study was also supported by the Emory Winship Research Pathology Core Lab.

Author contributions GJB, TC, JGM, STW, and DW designed the research. TPR, RHP, and RMP performed research with help from GMG, SMG, UAK, RMP, TM, and JPS. MPE, TPR, and RMP analyzed data. TC, JGM, TPR, and DW wrote the manuscript. All authors provided edits and approved final manuscript.

\section{Compliance with ethical standards}

Conflict of interest The authors declare that they have no conflict of interest.

Publisher's note: Springer Nature remains neutral with regard to jurisdictional claims in published maps and institutional affiliations.

\section{References}

1. Ballif BC, Theisen A, Coppinger J, Gowans GC, Hersh JH, Madan-Khetarpal S, et al. Expanding the clinical phenotype of the 3 q29 microdeletion syndrome and characterization of the reciprocal microduplication. Mol Cytogenet. 2008;1:8.

2. Glassford MR, Rosenfeld JA, Freedman AA, Zwick ME, Mulle JG, Unique Rare Chromosome Disorder Support G. Novel features of 3q29 deletion syndrome: results from the 3q29 registry. Am J Med Genet A. 2016;170A:999-1006.

3. Marshall CR, Howrigan DP, Merico D, Thiruvahindrapuram B, $\mathrm{Wu} \mathrm{W}$, Greer DS, et al. Contribution of copy number variants to schizophrenia from a genome-wide study of 41,321 subjects. Nat Genet. 2017;49:27-35.

4. Mulle JG, Dodd AF, McGrath JA, Wolyniec PS, Mitchell AA, Shetty AC, et al. Microdeletions of 3q29 confer high risk for schizophrenia. Am J Hum Genet. 2010;87:229-36. 
5. Mulle JG, Gambello MJ, Cook EH, Rutkowski TP, Glassford M. 3q29 recurrent deletion. In: Adam MP, Ardinger HH, Pagon RA, Wallace SE, Bean LJH, Stephens K, et al., editors. GeneReviews((R)). Seattle, WA; University of Washington, Seattle; 1993-2019. Available from: https://www.ncbi.nlm.nih.gov/ books/NBK385289/.

6. Sanders SJ, He X, Willsey AJ, Ercan-Sencicek AG, Samocha KE, Cicek AE, et al. Insights into autism spectrum disorder genomic architecture and biology from 71 risk loci. Neuron. 2015;87:1215-33.

7. Willatt L, Cox J, Barber J, Cabanas ED, Collins A, Donnai D, et al. 3q29 microdeletion syndrome: clinical and molecular characterization of a new syndrome. Am J Hum Genet. 2005;77:154-60.

8. Grozeva D, Conrad DF, Barnes CP, Hurles M, Owen MJ, O'Donovan MC, et al. Independent estimation of the frequency of rare CNVs in the UK population confirms their role in schizophrenia. Schizophr Res. 2012;135:1-7.

9. Mulle JG. The $3 q 29$ deletion confers $>40$-fold increase in risk for schizophrenia. Mol Psychiatry. 2015;20:1028-9.

10. Korablev AN, Serova IA, Serov OL. Generation of megabasescale deletions, inversions and duplications involving the Contactin- 6 gene in mice by CRISPR/Cas9 technology. BMC Genet. 2017;18(Suppl 1):112.

11. Carroll LS, Williams HJ, Walters J, Kirov G, O'Donovan MC, Owen MJ. Mutation screening of the 3q29 microdeletion syndrome candidate genes DLG1 and PAK2 in schizophrenia. Am J Med Genet B Neuropsychiatr Genet. 2011;156B:844-9.

12. Rutkowski TP, Schroeder JP, Gafford GM, Warren ST, Weinshenker D, Caspary T, et al. Unraveling the genetic architecture of copy number variants associated with schizophrenia and other neuropsychiatric disorders. J Neurosci Res. 2017;95:1144-60.

13. Leonard AS, Davare MA, Horne MC, Garner CC, Hell JW. SAP97 is associated with the alpha-amino-3-hydroxy-5-methylisoxazole-4-propionic acid receptor GluR1 subunit. J Biol Chem. 1998:273:19518-24.

14. Purcell SM, Moran JL, Fromer M, Ruderfer D, Solovieff N, Roussos $\mathrm{P}$, et al. A polygenic burden of rare disruptive mutations in schizophrenia. Nature. 2014;506:185-90.

15. Uezato A, Kimura-Sato J, Yamamoto N, Iijima Y, Kunugi H, Nishikawa T. Further evidence for a male-selective genetic association of synapse-associated protein 97 (SAP97) gene with schizophrenia. Behav Brain Funct. 2012;8:2.

16. Toyooka K, Iritani S, Makifuchi T, Shirakawa O, Kitamura N, Maeda K, et al. Selective reduction of a PDZ protein, SAP-97, in the prefrontal cortex of patients with chronic schizophrenia. J Neurochem. 2002;83:797-806.

17. Gupta P, Uner OE, Nayak S, Grant GR, Kalb RG. SAP97 regulates behavior and expression of schizophrenia risk enriched gene sets in mouse hippocampus. PLoS ONE. 2018;13:e0200477.

18. Wang Y, Zeng C, Li J, Zhou Z, Ju X, Xia S, et al. PAK2 haploinsufficiency results in synaptic cytoskeleton impairment and autism-related behavior. Cell Rep. 2018;24:2029-41.

19. Grice SJ, Liu JL, Webber C. Synergistic interactions between Drosophila orthologues of genes spanned by de novo human CNVs support multiple-hit models of autism. PLoS Genet. 2015;11:e1004998.

20. Mahoney ZX, Sammut B, Xavier RJ, Cunningham J, Go G, Brim $\mathrm{KL}$, et al. Discs-large homolog 1 regulates smooth muscle orientation in the mouse ureter. Proc Natl Acad Sci USA. 2006;103:19872-7.

21. Yang M, Silverman JL, Crawley JN. Automated three-chambered social approach task for mice. Curr Protoc Neurosci. 2011; Chapter 8: Unit 826.
22. Chalermpalanupap T, Schroeder JP, Rorabaugh JM, Liles LC, Lah JJ, Levey AI, et al. Locus coeruleus ablation exacerbates cognitive deficits, neuropathology, and lethality in P301S tau transgenic mice. J Neurosci. 2018;38:74-92.

23. Weinshenker D, Miller NS, Blizinsky K, Laughlin ML, Palmiter RD. Mice with chronic norepinephrine deficiency resemble amphetamine-sensitized animals. Proc Natl Acad Sci USA. 2002;99:13873-7.

24. R-CoreTeam. A language and environment for statistical computing. Vienna, Austria: R foundation for Statistical Computing; 2017. http://www.R-project.org.

25. Bates D, Machler M, Bolker BM, Walker SC. Fitting linear mixed-effects models using lme4. J Stat Softw. 2015;67:1-48.

26. Kuznetsova A, Brockhoff PB, Christensen RHB. lmerTest package: tests in linear mixed effects models. J Stat Softw. 2017;82:1-26.

27. Cox DM, Butler MG. A clinical case report and literature review of the 3q29 microdeletion syndrome. Clin Dysmorphol. 2015;24:89-94.

28. Nielsen J, Fejgin K, Sotty F, Nielsen V, Mork A, Christoffersen $\mathrm{CT}$, et al. A mouse model of the schizophrenia-associated 1q21.1 microdeletion syndrome exhibits altered mesolimbic dopamine transmission. Transl Psychiatry. 2017;7:1261.

29. Takahashi H, Nakamura T, Kim J, Kikuchi H, Nakahachi T, Ishitobi $\mathrm{M}$, et al. Acoustic hyper-reactivity and negatively skewed locomotor activity in children with autism spectrum disorders: an exploratory study. Front Psychiatry. 2018;9:355.

30. Kesby JP, Eyles DW, McGrath JJ, Scott JG. Dopamine, psychosis and schizophrenia: the widening gap between basic and clinical neuroscience. Transl Psychiatry. 2018;8:30.

31. Paval D. A dopamine hypothesis of autism spectrum disorder. Dev Neurosci. 2017;39:355-60.

32. Kidd JM, Cooper GM, Donahue WF, Hayden HS, Sampas N, Graves $\mathrm{T}$, et al. Mapping and sequencing of structural variation from eight human genomes. Nature. 2008;453:56-64.

33. Logue SF, Paylor R, Wehner JM. Hippocampal lesions cause learning deficits in inbred mice in the Morris water maze and conditioned-fear task. Behav Neurosci. 1997;111:104-13.

34. Morris RG, Hagan JJ, Rawlins JN. Allocentric spatial learning by hippocampectomised rats: a further test of the "spatial mapping" and "working memory" theories of hippocampal function. Q J Exp Psychol B. 1986;38:365-95.

35. Baez-Mendoza R, Schultz W. The role of the striatum in social behavior. Front Neurosci. 2013;7:233.

36. Koch M. The neurobiology of startle. Prog Neurobiol. 1999;59:107-28.

37. Murphy MM, Lindsey Burrell T, Cubells JF, Espana RA, Gambello MJ, Goines KCB, et al. Study protocol for The Emory 3q29 Project: evaluation of neurodevelopmental, psychiatric, and medical symptoms in 3q29 deletion syndrome. BMC Psychiatry. 2018;18:183.

38. Halladay AK, Bishop S, Constantino JN, Daniels AM, Koenig K, Palmer K, et al. Sex and gender differences in autism spectrum disorder: summarizing evidence gaps and identifying emerging areas of priority. Mol Autism. 2015;6:36.

39. Kirkovski M, Enticott PG, Fitzgerald PB. A review of the role of female gender in autism spectrum disorders. J Autism Dev Disord. 2013;43:2584-603.

40. Lai MC, Lombardo MV, Baron-Cohen S. Autism. Lancet. 2014;383:896-910.

41. Prendergast BJ, Onishi KG, Zucker I. Female mice liberated for inclusion in neuroscience and biomedical research. Neurosci Biobehav Rev. 2014;40:1-5. 